\title{
PAPERS
}

\section{One week triple therapy for Helicobacter pylori: a multicentre comparative study}

\begin{abstract}
J J Misiewicz, A W Harris, K D Bardhan, S Levi, C O'Morain, B T Cooper, G D Kerr,
\end{abstract} M F Dixon, H Langworthy, D Piper, Lansoprazole Helicobacter Study Group studied. LAC was the most efficacious treatment in patients with pretreatment metronidazole resistant $H$ pylori, and was significantly better than LAM and OAM in this group of patients.

(Gut 1997; 41: 735-739) tritis.

Aim-To compare four treatment regimens lasting seven days of a proton pump inhibitor and two antibiotics in the eradication of $\boldsymbol{H}$ pylori.

Patients-Men or women with $H$ pylori positive duodenal ulceration or gastritis, or both.

Central Middlesex

Hospital,

London

J J Misiewicz

A W Harris

Rotherham District Hospital

K D Bardhan

Northwick Park

Hospital,

Slough

S Levi

The Meath Hospital, Dublin

C O’Morain

Royal Hospital, Birmingham

B T Cooper

Royal Shrewsbury

Hospital

G D Kerr

General Infirmary, Leeds

M F Dixon

Lederle Laboratories, Gosport

H Langworthy

D Piper

Correspondence to: Dr J J Misiewicz,

Department of

Gastroenterology and

Nutrition, Central Middlesex

Hospital NHS Trust, Park

Royal, London NW1D 7NS,

UK.

Accepted for publication

7 August 1997 randomised, parallel group, comparative, multicentre study. After a positive CLO test, patients underwent histology, $H$ pylori culture, and $a^{13} \mathrm{C}$ urea breath test to confirm $H$ pylori status. Treatment with one of four regimens: LAC, LAM, LCM, or $O A M$, where $L$ is $30 \mathrm{mg}$ of lansoprazole twice daily, $A$ is $1 \mathrm{~g}$ of amoxycillin twice daily, $M$ is $400 \mathrm{mg}$ of metronidazole twice daily, $C$ is $250 \mathrm{mg}$ of clarithromycin twice daily, and $O$ is $20 \mathrm{mg}$ of omeprazole twice daily, was assigned randomly. A follow up breath test was done at least 28 days after completing treatment.

Results-H pylori eradication (intention to treat) was $104 / 121(86.0 \%)$ with LAC, 87/131 (66.4\%) with LAM, 103/118 (87.3\%) with LCM, and 94/126 (74.6\%) with OAM. There was a significant difference ( $p<$ 0.001 ) in the proportion of patients in whom eradication was successful between LAC and LCM when compared with LAM, but no significant difference $(p=0.15)$ between LAM and OAM. Metronidazole resistance before treatment was identified as a significant prognostic factor with regard to eradication of $H$ pylori. The regimens which contained metronidazole were significantly less effective than those without metronidazole in the presence of pretreatment resistant $H$ pylori. There was no difference among the treatment groups with regard to the incidence and severity of adverse events reported.

Conclusions-All four treatment regimens were safe and effective in eradicating $H$ pylori in the patient population
Methods-A single blind, prospectively
Keywords: eradication; Helicobacter pylori; lansoprazole; omeprazole; metronidazole resistance

Helicobacter pylori is recognised to be the main aetiological factor in the pathogenesis of duodenal ulcer and non-autoimmune gastritis. Approximately $95 \%$ of patients with duodenal dyspepsia are colonised with $\mathrm{H}$ pylori. ${ }^{12}$ Several studies have shown that eradication of $H$ pylori cures duodenal ulcer and prevents relapse. ${ }^{3}$ In addition, after eradication of $H$ pylori there is histological resolution of chronic active gastritis. $^{4}$ cation of $H$ pylori have been widely sought, and the treatment currently recommended by the National Institute of Health Consensus is bismuth, tetracycline, and metronidazole, all given for 14 days. $^{5}$ A high incidence of side effects with this treatment, however, has encouraged researchers to identify regimens which are as effective, less complex, with fewer side effects, and of shorter duration, thereby encouraging compliance. The most successful eradication regimens to date have been one week treatments with a proton pump inhibitor and two antibiotics. ${ }^{6-8}$

The aim of this study was to investigate the efficacy and safety of lansoprazole in combination with two antibiotics, all given twice daily for seven days, in the eradication of $H$ pylori. In addition, since there have been no direct comparative studies of the two proton pump inhibitors, a treatment arm containing omeprazole and two antibiotics was included. Furthermore, the efficacy of the four treatment regimens against pretreatment metronidazole resistant strains of $H$ pylori was assessed.

\section{Patients and Methods}

Patients of either sex aged between 18-80 years inclusive, with a duodenal ulcer or gastritis, or both, who were $H$ pylori positive (as determined by a CLO test (Delta West, Bentley, West ulcer and $50 \%$ of patients with non-ulcer

Different treatment regimens for the eradi- 
Australia) at endoscopy) were invited to undergo a ${ }^{13} \mathrm{C}$ urea breath test (Bureau of Stable Isotope Analysis, Brentford, UK) to confirm $H$ pylori status. All patients presenting between November 1994 and May 1995 were invited to enter the study if they fulfilled the entry criteria and were able to sign a consent form. Any patients who had a negative ${ }^{13} \mathrm{C}$ urea breath test were not eligible to enter the study. Other exclusion criteria were treatment with proton pump inhibitors, compounds containing bismuth, sucralfate or antibiotics within the two weeks before study entry, and allergy to any of the study drugs. Patients were not permitted to take $\mathrm{H}_{2}$ receptor antagonists or any other ulcer healing drugs while receiving study medication. Additionally, patients were excluded if they had already participated in the study, were participating in another study, or had significant gastrointestinal, renal, hepatic, cardiovascular, metabolic, or haematological disease.

The study consisted of a single blind, prospectively randomised, parallel group, comparative multicentre study carried out between November 1994 and May 1995 in 55 hospitals in the UK and Ireland.

The study was approved by the local Ethics Committee at each participating centre and written informed consent was obtained from patients before entering the study.

DETERMINATION OF H PYLORI STATUS

A single antral biopsy specimen was taken for the CLO test, and a further six biopsy specimens were taken at the baseline endoscopy for histological examination (two antral and two corpus) and microbiological culture (two antral). Successful cultures were grown and tested at a central laboratory using $\mathrm{E}$ test strips for sensitivity to metronidazole, clarithromycin, and amoxycillin. Resistance to metronidazole, clarithromycin, and amoxycillin was defined as minimal inhibitory concentration of $\geqslant 8 \mu \mathrm{g} / \mathrm{ml}, \geqslant 0.1 \mu \mathrm{g} / \mathrm{ml}$, and $\geqslant 0.25$ $\mu \mathrm{g} / \mathrm{ml}$ respectively. A single histopathologist (MFD), who was unaware of the identification of the patients, reported the histology, which was graded on an ordinal scale for the presence of $H$ pylori $(0=$ no bacteria present, $1=$ occasional bacteria present, not in every field, $2=$ moderate numbers of organisms seen in most fields, 3 = numerous bacteria seen in all fields). Histological evidence of gastritis was recorded as present or absent. All patients underwent a ${ }^{13} \mathrm{C}$ urea breath test according to the European protocol within seven days of the endoscopy to confirm $H$ pylori status. ${ }^{9}$ An excess value of 5.0 $\delta^{13} \mathrm{C}$ or greater was considered as a positive result. Patients were only eligible for the study if they had a positive breath test and at least one positive result from histopathology or microbiological assessment of $H$ pylori.

TREATMENT

Patients were randomly allocated to one of the following treatment regimens by a computer generated randomisation list stratified by centre in blocks of four patients: $30 \mathrm{mg}$ of lansoprazole twice daily, $1 \mathrm{~g}$ of amoxycillin twice daily, $250 \mathrm{mg}$ of clarithromycin twice daily (LAC); $30 \mathrm{mg}$ of lansoprazole twice daily,
$1 \mathrm{~g}$ of amoxycillin twice daily, $400 \mathrm{mg}$ of metronidazole twice daily (LAM); $30 \mathrm{mg}$ of lansoprazole twice daily, $250 \mathrm{mg}$ of clarithromycin twice daily, $400 \mathrm{mg}$ of metronidazole twice daily (LCM); and $20 \mathrm{mg}$ of omeprazole twice daily, $1 \mathrm{~g}$ of amoxycillin twice daily, $400 \mathrm{mg}$ of metronidazole twice daily (OAM).

Treatment was dispensed in seven day blister packs. No further treatment was given. Each blister contained all three drugs to be taken at each dosing time. All medication was taken each day, 30 minutes before breakfast and the evening meal.

\section{ASSESSMENTS}

Patients returned for a clinic visit (visit 2) as soon as they had completed study treatment, and again at least 28 days later (visit 3). At visit 2 and visit 3 , patients were questioned regarding the incidence of any adverse events. At visit 3, all patients underwent a ${ }^{13} \mathrm{C}$ urea breath test. Endoscopy was done on all patients found to have a positive breath test at visit 3, to enable the determination of $H$ pylori culture and sensitivity in patients for whom treatment had failed.

\section{STATISTICS}

The primary measure of efficacy was the eradication of $H$ pylori as determined by the results of ${ }^{13} \mathrm{C}$ urea breath test at visit 3 . We calculated that 112 patients per treatment arm would be required to show equivalence between LAM and OAM (assuming $80 \%$ of patients treated would have their $H$ pylori eradicated) within $15 \%$ with a power of $80 \%$ at the $5 \%$ level of significance (two-tailed).

The per protocol and intention to treat populations were included in the efficacy analyses. The per protocol population included only those eligible patients who had undergone a urea breath test at least 28 days after completion of treatment and had taken at least $70 \%$ of the study medication. The intention to treat population (the primary efficacy population) included all patients who had taken at least one dose of study medication. It was assumed that $H$ pylori had not been eradicated if the patient did not return for a breath test at visit 3, that is, a worst case analysis. All patients who took the treatment, regardless of their eligibility for the study, were included in the safety analyses, which compared the treatments with respect to the maximum incidence and severity of the reported clinical adverse events using the $\chi^{2}$ and Kruskal-Wallis tests, respectively.

Treatment groups were compared with respect to the proportion of patients in whom eradication was successful using a $\chi^{2}$ test on three degrees of freedom. If a statistically significant difference was detected, pairwise comparisons were carried out, using the Fisher's exact test and the Bonferroni correction. Values of $p<0.05$ were considered significant. The effect of pretreatment potential prognostic factors on the proportion of patients in whom $H$ pylori was eradicated were investigated using logistic regression analysis. Odds ratios were estimated together with $95 \%$ confidence intervals (OR (95\% CI)). 
TABLE 1 Pretreatment clinical and demographic characteristics

\begin{tabular}{lllll}
\hline \multicolumn{4}{l}{ Treatment group } \\
\cline { 2 - 5 } & $\begin{array}{l}\text { LAC } \\
(n=121)\end{array}$ & $\begin{array}{l}\text { LAM } \\
(n=131)\end{array}$ & $\begin{array}{l}\text { LCM } \\
(n=118)\end{array}$ & $\begin{array}{l}\text { OAM } \\
(n=126)\end{array}$ \\
\hline $\begin{array}{l}\text { Mean age (years) } \\
\quad \text { Range }\end{array}$ & 48.1 & 47.6 & 49.1 & 48.0 \\
$\begin{array}{l}\text { Sex } \\
\quad \text { Men (\%) }\end{array}$ & $22-78$ & $20-80$ & $23-77$ & $21-74$ \\
$\quad$ Women (\%) & $82(68)$ & $94(72)$ & $72(61)$ & $88(70)$ \\
$\begin{array}{l}\text { Diagnosis } \\
\text { Duodenal ulcer (\%) }\end{array}$ & $39(32)$ & $37(28)$ & $46(39)$ & $38(30)$ \\
$\quad$ Gastritis (\%) & $62(51)$ & $64(49)$ & $61(52)$ & $75(60)$ \\
\hline & $59(49)$ & $67(51)$ & $57(48)$ & $51(40)$ \\
\hline
\end{tabular}

\section{Results}

EFFICACY

Five hundred and eight patients were recruited from 47 centres. The median number of patients entered per centre was eight (range 1-71). Twelve patients were ineligible to enter the study due to negative, or inconclusive, baseline breath test results. Table 1 shows baseline characteristics of the 496 eligible patients. Of these 496 patients, 443 were eligible for inclusion in the per protocol analysis. Most of the 53 patients who were excluded had either undergone their breath test less than 28 days after completion of treatment, or had not attended visit 3 .

Table 2 shows the proportion of patients in whom $H$ pylori was successfully eradicated for the per protocol and the intention to treat patient populations. Pairwise analysis showed that LCM and LAC were significantly better than LAM (Fisher's exact test $\mathrm{p}<0.001$ ) in both patient populations. There was no significant difference between OAM and LAM ( $p=$ 0.15 ) or between any of the other possible pairwise comparisons.

Logistic regression analysis was used to assess the effect of potential prognostic factors on the eradication of $H$ pylori. Patient's sex, age, smoking status, alcohol consumption, diagnosis (duodenal ulcer $v$ gastritis), treatment with $\mathrm{H}_{2}$ receptor antagonists in the month before study entry, or the duration of the patient's disease had no significant effect ( $\mathrm{p}$ $>0.05$ ) on the eradication of $H$ pylori.

EFFECT OF PRETREATMENT METRONIDAZOLE RESISTANCE

Culture of $H$ pylori was successful in 446 patients $(89.9 \%)$, and metronidazole sensitivity was reported for 438 of these cultures. Table 3 summarises the pretreatment metronidazole sensitivities and the proportion of patients in whom $H$ pylori was eradicated for patients with pretreatment metronidazole sensitive, and for those with pretreatment metronidazole resistant strains, for both patient populations. Con-

TABLE 2 Proportion of patients with negative ${ }^{13} \mathrm{C}$ urea breath test 28 days after treatment

\begin{tabular}{lllll}
\hline \multirow{5}{*}{ Breath test result } & Treatment group & & \\
\cline { 2 - 5 } & $L A C$ & $L A M$ & $L C M$ & OAM \\
\hline Intention to treat & $(\mathrm{n}=121)$ & $(\mathrm{n}=131)$ & $(\mathrm{n}=118)$ & $(\mathrm{n}=126)$ \\
$\quad$ Negative $(\%)$ & $104(86.0)$ & $87(66.4)$ & $103(87.3)$ & $94(74.6)$ \\
$95 \%$ CI & $(82.3,94.3)$ & $(63.5,80.1)$ & $(83.0,94.8)$ & $(73.2,88.1)$ \\
Per protocol & $(\mathrm{n}=114)$ & $(\mathrm{n}=113)$ & $(\mathrm{n}=109)$ & $(\mathrm{n}=107)$ \\
Negative $(\%)$ & $103(90.4)$ & $83(73.5)$ & $99(90.8)$ & $89(83.2)$ \\
$95 \%$ CI & $(83.0,94.8)$ & $(64.2,81.1)$ & $(83.4,95.3)$ & $(74.4,89.5)$ \\
\hline
\end{tabular}

sistent results were observed for the intention to treat and the per protocol populations. Three of the four treatment groups (LAM, LCM, and OAM) showed greater $H$ pylori eradication rates for patients who had pretreatment metronidazole sensitive $H$ pylori compared with those who had pretreatment resistant $H$ pylori. For the LAC treatment group, similar eradication rates were observed regardless of whether the $H$ pylor $i$ was metronidazole resistant or sensitive. Metronidazole resistance had no effect on the eradication of $H$ pylori for patients receiving treatment with LAC as shown by odds ratios (that is, the odds of $H$ pylori eradication for a patient with metronidazole sensitive $H$ pylori compared with a patient with metronidazole resistant $H$ pylori) of 0.64 $(0.16,2.65)$, but the odds ratios for LAM, LCM, and OAM were $11.08(3.88,33.15)$, $5.45(1.39,21.30)$, and $7.11(1.96,25.71)$, respectively. LAC was significantly better than both LAM and OAM ( $\mathrm{p}=0.001$ and 0.02 respectively) in eradicating $H$ pylori in patients with pretreatment metronidazole resistant organisms (per protocol population) although there was no significant difference between LAC and LCM ( $p=0.08$, Fisher's exact test), nor between LAM and OAM ( $p=0.09$, Fisher's exact test). Similar results were observed in the intention to treat population as LAC was significantly better than LAM and OAM ( $p<$ 0.001 and $p=0.003$ respectively), but not superior to LCM ( $\mathrm{p}=0.14)$.

Two of the seven patients in the LAC group with pretreatment metronidazole sensitive strains in whom eradication was not successful had clarithromycin minimal inhibitory concentration values of $\geqslant 3 \mu \mathrm{g} / \mathrm{ml}$ before treatment, suggesting primary resistance to clarithromycin. In the LAM group, four of the six patients with pretreatment metronidazole sensitive $H$ pylori in whom eradication had not been successful appeared to have acquired metronidazole resistant $H$ pylori by visit 3, with no sensitivity data available for the remaining two patients. Similarly, two patients in the LCM group had metronidazole resistant $H$ pylori at visit 3, and one patient had developed clarithromycin resistant $H$ pylori at visit 3. Two of the four patients in the OAM group in whom eradication had been unsuccessful had metronidazole resistant $H$ pylori at visit 3; in the remaining two patients sensitivity data were not available.

SAFETY

The safety analyses were done on all 508 patients who had taken at least one dose of study medication. A total of 458 adverse events were reported by $271(53.3 \%)$ of the 508 treated patients during the study (table 4). There was no difference among the treatment groups with respect to the incidence $\left(\chi^{2}=1.73\right.$, degrees of freedom $=3, p=0.63)$, or severity of adverse events (Kruskal-Wallis test, $\chi^{2}=0.94$, degrees of freedom $=3, p=0.81$ ).

The most frequently reported adverse events were diarrhoea, headache, and taste disturbance. Fifty six patients experienced diarrhoea $(\mathrm{LAC}=22, \mathrm{LAM}=12, \mathrm{LCM}=5, \mathrm{OAM}=7)$, 34 patients reported headache (LAC $=7$, LAM 
TABLE 3 Pretreatment metronidazole sensitivity and eradication of $\mathrm{H}$ pylori

\begin{tabular}{|c|c|c|c|c|}
\hline & \multicolumn{4}{|c|}{ Treatment group } \\
\hline & $L A C$ & $L A M$ & $L C M$ & $O A M$ \\
\hline \multicolumn{5}{|c|}{ Pretreatment metronidazole sensitivity } \\
\hline \multicolumn{5}{|c|}{ Intention to treat ${ }^{\star}$} \\
\hline Resistant (\%) & $39(38.2)$ & $41(37.6)$ & $28(27.2)$ & $32(32.7)$ \\
\hline Sensitive (\%) & $63(61.8)$ & $68(62.4)$ & $75(72.8)$ & $66(67.3)$ \\
\hline \multicolumn{5}{|l|}{ Per protocol } \\
\hline Resistant (\%) & $39(39.0)$ & $39(38.2)$ & $25(25.5)$ & $28(30.4)$ \\
\hline Sensitive (\%) & $62(61.0)$ & $63(61.8)$ & $73(74.5)$ & $64(69.6)$ \\
\hline \multicolumn{5}{|c|}{ Eradication by metronidazole sensitivity } \\
\hline \multicolumn{5}{|c|}{ Intention to treat ${ }^{\star}$} \\
\hline Resistant (\%) & $36 / 39(92.3)$ & $19 / 41(46.3)$ & $21 / 28(75.0)$ & $20 / 32(62.5)$ \\
\hline$(95 \% \mathrm{CI})$ & $(78.0,98.0)$ & $(31.0,62.4)$ & $(54.8,88.6)$ & $(43.7,78.3)$ \\
\hline Sensitive (\%) & $55 / 63(87.3)$ & $60 / 68(88.2)$ & $71 / 75(94.7)$ & $62 / 66(93.9)$ \\
\hline$(95 \% \mathrm{CI})$ & $(76.0,94.0)$ & $(77.6,94.4)$ & $(86.2,98.3)$ & $(84.4,98.0)$ \\
\hline \multicolumn{5}{|l|}{ Per protocol } \\
\hline Resistant (\%) & $36 / 39(92.3)$ & $18 / 39(46.2)$ & $19 / 25(76.0)$ & $19 / 28(67.9)$ \\
\hline$(95 \% \mathrm{CI})$ & $(78.0,98.0)$ & $(30.4,62.6)$ & $(54.5,89.8)$ & $(47.6,83.4)$ \\
\hline Sensitive (\%) & $54 / 61(88.5)$ & $57 / 63(90.5)$ & $69 / 73(94.5)$ & $60 / 64(93.8)$ \\
\hline$(95 \% \mathrm{CI})$ & $(77.2,94.9)$ & $(79.8,96.1)$ & $(85.8,98.2)$ & $(84.0,98.0)$ \\
\hline
\end{tabular}

$\star$ This is based on those patients in the intention to treat population $(n=496)$ who had returned for a visit 3 breath test $(n=465)$ minus those patients $(n=53)$ who had no pretreatment metronidazole sensitivity reported $(n=412)$.

TABLE 4 Number of adverse events reported per patient

\begin{tabular}{lrrrr}
\hline \multirow{4}{*}{ No of adverse events } & Treatment group & \\
\cline { 2 - 5 } & LAC & LAM & LCM & OAM \\
\hline 0 & 64 & 57 & 58 & 58 \\
1 & 36 & 42 & 34 & 39 \\
2 & 15 & 16 & 16 & 21 \\
3 & 6 & 15 & 10 & 7 \\
4 & 5 & 2 & 3 & 3 \\
5 & 0 & 0 & 0 & 1 \\
Total & 126 & 132 & 121 & 129 \\
\hline
\end{tabular}

$=10, \mathrm{LCM}=11, \mathrm{OAM}=6$ ), and 26 patients reported some form of taste disturbance (LAC $=2, \mathrm{LAM}=7, \mathrm{LCM}=11, \mathrm{OAM}=6$ ).

Seven patients $(\mathrm{LAC}=2, \mathrm{LAM}=1, \mathrm{OAM}=$ 4) withdrew from the study as a result of an adverse event, none of which was considered to be clinically serious. Five patients reported serious adverse events, four of which (pancreatic carcinoma, abnormal ECG, oesophageal carcinoma, and arteriosclerotic heart disease) were considered by the treating clinician as definitely not related to study treatment. One patient who was given OAM developed pseudomembranous colitis which was successfully treated with vancomycn.

\section{Discussion}

This study has shown that a one week combination of a proton pump inhibitor with two antibiotics effectively eradicates $H$ pylor $i$ in patients with duodenal ulcer or non-ulcer gastritis, or both. The proportion of patients in whom $H$ pylori was eradicated was comparable with that reported by others who have investigated similar drug combinations. ${ }^{6-8}$ All treatments were sufficiently efficacious to be considered clinically useful.

The influence of pretreatment $H$ pylori metronidazole sensitivity on the eradication efficacy of a treatment combination is highlighted by the significant difference in results in patients with pretreatment metronidazole resistant strains of $H$ pylori. Two of the three metronidazole containing regimens were significantly less effective at eradicating $H$ pylori infection in the presence of metronidazole resistance than the non-metronidazole con- taining regimen. By contrast, there was no significant difference in efficacy among the four treatments in metronidazole sensitive strains. It may therefore be important when considering treatment to prescribe according to the metronidazole sensitivity status of the individual patient's $H$ pylori, if known. Unfortunately this information is rarely available before starting treatment. This study suggests, however, that it is still possible to prescribe efficacious treatment regardless of metronidazole sensitivity status. The prevalence of pretreatment metronidazole resistant strains of $H$ pylori varies considerably from around $90 \%$ in urban/inner city areas to $22 \%$ in rural/provincial areas. $^{10}{ }^{11}$ Using the information from the intention to treat population in table 3 and the laws of probability, it can be shown that if the prevalence of metronidazole resistant $H$ pylori is at least $30 \%$ (ignoring sampling error), LAC should be the first choice of treatment.

While the eradication efficacy of LAM and OAM in this study is clinically acceptable, comparison with other reports suggests that the proportion of patients in whom $H$ pylori is eradicated is higher when antibiotics are given three times, rather than twice daily. ${ }^{12-14}$ The results of this study suggest that if patients are known to have a strain of $H$ pylori that is metronidazole sensitive, giving twice daily doses of the antibiotics results in equivalent eradication efficacies to the two clarithromycin regimens. If patients have a metronidazole resistant strain of $H$ pylori, however (or the sensitivity is not known or cannot be ascertained), it is justifiable to increase the frequency of dosing of the antibiotics in order to increase the proportion of patients in whom eradication will be successful, or use LAC given twice daily.

A large multicentre study of similar, but omeprazole based, regimens reported successful eradication of $\mathrm{H}$ pylori in 105 of 111 (95\%) of patients using OCM given at the same dosing schedule as LCM in this study. ${ }^{7}$ Data concerning the pretreatment antimicrobial sensitivities of $H$ pylori in the omeprazole study are not available. It may be that this higher (per protocol) result could depend on a lower 
prevalence of metronidazole resistant strains than in this study.

On the other hand, the importance of metronidazole resistance as a major factor in determining the outcome of eradication regimens for $H$ pylori is perhaps not as clear as previously thought. Two recent studies have not shown any effect of metronidazole resistance on the success of treatment with regimens that include metronidazole. ${ }^{16}{ }^{17}$ One study used the LCM regimen, but the dose of lansoprazole was $30 \mathrm{mg}$ daily and not twice daily as in the present investigation. ${ }^{16}$ In another multicentre study, pretreatment metronidazole sensitivity apparently had no effect on the outcome of twice daily treatment with omeprazole, clarithromycin, and metronidazole (OCM) or with bismuth subcitrate, clarithromycin, and metronidazole (BCM) ${ }^{17}$ However, a regimen consisting of omeprazole, amoxycillin, and metronidazole (OAM) was just significantly less effective in patients colonised by metronidazole resistant strains of $H$ pylori. It is noteworthy that in the present study the advantage of LAC in metronidazole resistant strains of $H$ pylori was quite definite, that is, $\mathrm{p}=0.001$ compared with LAM, and 0.02 compared with OAM. These conflicting data cannot be resolved at present and further large scale studies will be needed to provide an answer. In the meantime, however, the LAC regimen provides a metronidazole free treatment with a $90 \%$ chance of success.

While the number of patients reporting adverse events $(53.3 \%)$ was relatively high compared with other studies, ${ }^{6-81213}$ there was no overall difference among the treatment groups with regard to the number of events reported. It should be noted, however, that the adverse events recorded in this study were all events reported by patients, regardless of drug attribution by the clinician. In this study, particular attention was paid to side effects, and patients were directly asked whether they had experienced any unusual symptoms during or after treatment. It is possible that the high reported prevalence of side effects may reflect this approach. Only a very small proportion of patients $(1.4 \%)$ withdrew from the study because of side effects, which was in keeping with other reports..$^{6-81213}$ The treatments were thus reasonably well tolerated. The high incidence of taste related adverse events is a frequently reported occurrence with clarithromycin and metronidazole, but no patients withdrew from the study because of this. Diarrhoea or gastrointestinal disturbance is also a well documented side effect of the five drugs used in this study, thus the relatively large number of patients reporting diarrhoea is consistent with current knowledge of treatment with these drugs.

In conclusion, all four treatment regimens investigated in this study were safe and efficacious in the eradication of $H$ pylori. In the presence of pretreatment metronidazole sensitive strains of $H$ pylori the treatments were of similar efficacy. In areas of high prevalence of metronidazole resistant strains of $H$ pylori, however, a regimen based on a proton pump inhibitor with amoxycillin and clarithromycin
- LAC in this study — could be considered as first line treatment.

Previously presented in part on March 20th at the 1996 Spring Meeting of the British Society of Gastroenterology, Brighton, UK. This work has been previously published as an abstract in Gut 1996;38:A1.

The authors would like to thank the following clinicians who entered patients into this study:

Dr M Allison, Royal Gwent Hospital, Newport; Dr ATR Axon, Leeds General Infirmary, Leeds; Dr A Beattie, Southern General Hospital, Glasgow; Dr D Bell, Ipswich Hospital, Ipswich; Dr P Cann, Middlesborough General Hospital, Middlesborough; Mr LR Celestin, St Mary's Hospital, Bristol; Middlesborough; Mr LR Celestin, St Mary's Hospital, Bristol; Dr R Chapman, John Radcliffe Hospital, Oxford; Dr I Chesner, Heartlands Hospital, Birmingham; Dr J Crowe, Mater MiseraGlasgow; Dr TK Daneshmend, Royal Devon and Exeter Glasgow; Dr TK Daneshmend, Royal Devon and Exeter Hospital, Exeter; Dr P Davies, East Glamorgan Hospital, Pon-
typridd; Dr ME Denyer, Seacroft Hospital, Leeds; Dr WR Ellis, typridd; Dr ME Denyer, Seacroft Hospital, Leeds; Dr WR Ellis,
Dryburn Hospital, Durham; Dr O Epstein, Royal Free Hospital, London; Dr PD Fairclough, St Bartholomew's Hospital, London; Dr D Fine, Southampton General Hospital, Southampton; Dr P Finch, St Peter's Hospital, Chertsey; Dr C Gilbertson, Nevill Hall Hospital, Abergavenny; Mr A Gough, Weston General Hospital, Weston Super Mare; Professor CJ Hawkey, University Hospital, Nottingham; Dr J Hunter, Addenbrookes Hospital, Cambridge; Mr G Hutchinston, Halton General Hospital, Runcorn; Dr PN Keeling, St James Hospital, Dublin; Mr R Kingston, Trafford General Hospital, Mosphester; Dr F Lee, Royal Victoria Infirm General Hospital, Manchester; Dr F Lee, Royal Victoria Infirmary, Blackpool; Dr RG Long, City Hospital, Nottingham; Dr V Mani, Leigh Infirmary, Leigh; Dr AS Mee, Battle Hospital, Reading; Dr JP Giller, Withington Hospital, Manchester; Dr P Mills, Gartnavel Infirmary, Bristol; Dr M Myszor, Royal Berkshire Hospital,
Reading; Dr M Panos, Royal Berkshire Hospital, Reading; Dr K Reading; Dr M Panos, Royal Berkshire Hospital, Reading; Dr K
Palmer, Western General Hospital, Edinburgh; Mr I Paterson, Palmer, Western General Hospital, Edinburgh; Mr I Paterson, Solihull; Dr C Pennington, Ninewells Hospital, Dundee; Dr DS Rampton, The Royal London Hospital, London; Dr JM Rhodes, Royal Liverpool University Hospital, Liverpool; Dr H Shepherd, Royal Hampshire County Hospital, Winchester; Dr G Tildesley, Hartlepool General Hospital, Hartlepool; Dr P Trewby, Memorial Hospital, Darlington; Mr C Venables, Freeman Hospital, Newcastle; Professor D Weir, St James Hospital, Dublin; Dr PJ Whorwell, Withington Hospital, Mancheser.

Supported by Lederle Laboratories, Gosport, UK.

1 Marshall BJ, McGechie DB, Rogers PA, Glancy RG. Pyloric camplylobacter infection and gastrointestinal disease. Med f Aust 1985;142:439-44.

2 Talley NJ. Is Helicobacter pylori a cause of non-ulcer dyspepsia? In: Malfertheiner P, Ditschumeit $\mathrm{H}$ eds. Helicobacter pylori, gastritis and peptic ulcer. Springer Verlag, Berlin 1990

3 Coghlan JG, Humphries H, Dooley C, et al. Campylobacter pylori and recurrence of duodenal ulcer - a 12 month follow up study. Lancet 1987 ;ii:1109-11.

4 Rauws EAJ, Langenberg W, Houthoff $\mathrm{HJ}$, et al. Campylobacter pyloridis-associated chronic antral gastritis. Gastroenterology 1988;94:33-40.

5 NIH Consensus Development Panel. Helicobacter pylori in peptic ulcer disease. $\mathcal{F A M A}$ 1994;272:65-9.

peptic ulcer disease. $\mathcal{F} A M A$ 1994;272:65-9.
6 Bazzoli F, Zagari RM, Fossi S, et al. Short term low dose triBazzoli $\mathrm{F}$, Zagari RM, Fossi S, et al. Short term low dose tri-
ple therapy for the eradication of Helicobacter pylori. Eur $\mathcal{f}$ ple therapy for the eradication of Helic
Gastroenterol Hepatol 1994;6:773-7.

7 Lind T, Veldhuyzen van Zanten SJO, Unge P, et al. The MACH 1 study: Optimal one-week treatment for H. pylori defined [abstract]? Gut 1995;37:A4

8 Labenz J, Stolte M, Peitz U, et al. One-week triple therapy with omeprazole, amoxycillin, and either clarithromycin or metronidazole for cure of Helicobacter pylori infection. Aliment Pharmacol Ther 1996;10:207-10.

9 Logan RPH, Dill S, Bauer FE, et al. The European 13C-urea breath test for the detection of $\mathrm{H}$. pylori. Eur $\mathcal{F}$ Gastroenterol Hepatol 1991;3:905-11.

10 Banatvala N, Davies GR, Abdi Y, et al. High prevalence of Helicobacter pylori metronidazole resistance in migrants to east London: relationship with previous nitroimidazole

1 Karim QN, Logan RPH. The epidemiology of Helicobacter pylori (H. pylori) antimicrobial resistance-preliminary pylori (H. pylori) antimicrobial resistance-preliminary
findings of a national survey [abstract]. Gut 1995;37(suppl 2):A6.

12 Bell GD, Powell KU, Burridge SM, et al. Rapid eradication of Helicobacter pylori infection. Aliment Pharmacol Ther 1995;9:41-6.

13 Bateson M, Diffey BL. Radical therapy for duodenal ulcer [abstract]. Gut 1995;36 (suppl 1):A35.

14 Harris AW, Misiewicz JJ. Eradication of H. pylori. Baillieres Clin Gastroenterol 1995;9:583-613.

15 Graham DY, Lew GM, Malaty HM, et al. Factors influencing the eradication of Helicobacter pylori with triple therapy. Gastroenterology 1992;102:493-6.

16 Pryce DI, Harris AW, Gabe S, et al. One week of lansoprazole, clarithromycin and metronidazole eradicates Helicozole, clarithromycin and metronidazole eradicates Helico-

17 Lerang F, Moum B, Haug JB, et al. Highly effective twice a day triple therapies for Helicobacter pylori infection and peptic ulcer disease-metronidazole resistance is of only minor importance [abstract]. Gastroenterology 1996;110: A173. 